\title{
Sub- and near-barrier fusion reactions experimental results
}

\author{
G. Montagnoli
}

Dipartimento di Fisica e Astronomia, Università di Padova, Padova, Italy and INFN, Sezione di Padova, Padova, Italy

\begin{abstract}
Early data of sub-barrier fusion teached us that cross sections may strongly depend on the structure of colliding nuclei and on couplings to transfer channels. The influence of transfer is clearly indicated in the excitation functions of different nickel isotopes and various $\mathrm{Ca}+\mathrm{Zr}$ systems. Fusion barrier distributions often yield the fingerprint of the relevant inelastic and transfer couplings. At lower energies, far below the barrier the slope of the excitation function keeps increasing in many cases, so that the cross sections are strongly over-predicted by standard coupled-channels (CC) calculations; this was named a hindrance effect.

Furthermore, light heavy-ion systems show cross section oscillations above the Coulomb barrier. Recent experiments have been performed on the fusion of ${ }^{28,30} \mathrm{Si}+{ }^{28,30} \mathrm{Si}$ systems where all phenomena cited above show up. In particular regular oscillations that have been revealed above the barrier for ${ }^{28} \mathrm{Si}+{ }^{28} \mathrm{Si}$ and have been interpreted as the consequence of the strong channel couplings and/or the oblate deformation of ${ }^{28} \mathrm{Si}$.
\end{abstract}




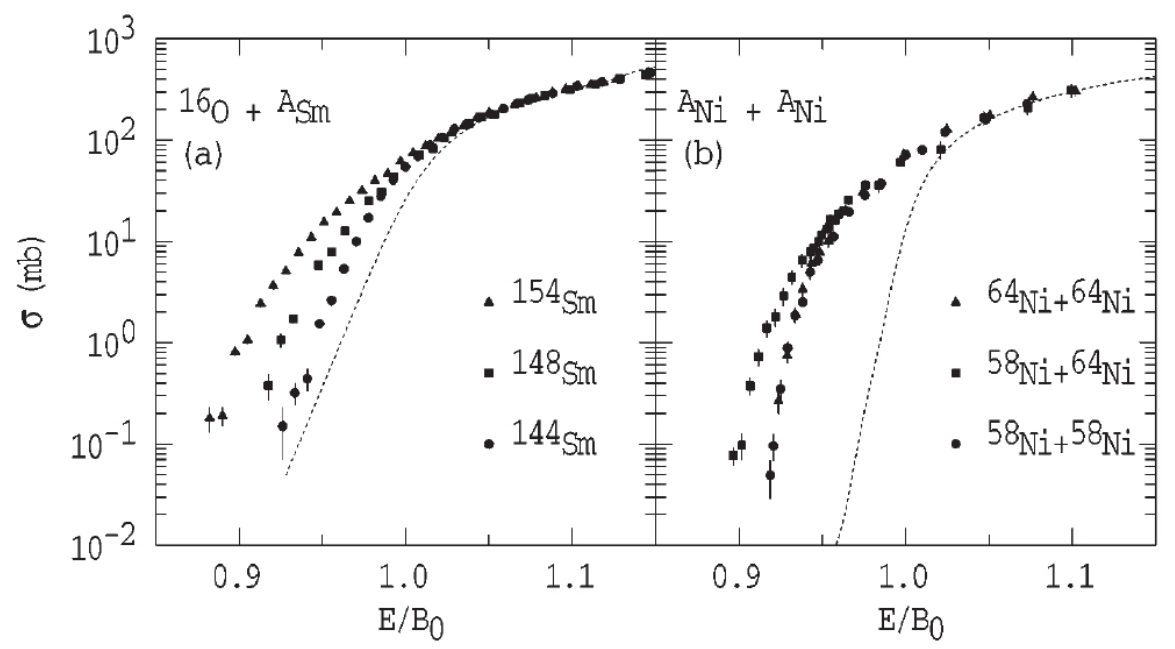

Figure 1: (a) Fusion excitation functions for ${ }^{16} \mathrm{O}$ on $\mathrm{Sm}$ isotopes [3] show a marked increase of cross section with increasing mass number and deformation. (b) The difference in the energy dependence of the cross sections [4] for the ${ }^{58} \mathrm{Ni}+{ }^{58} \mathrm{Ni}$, ${ }^{58} \mathrm{Ni}+{ }^{64} \mathrm{Ni}$, and ${ }^{64} \mathrm{Ni}+{ }^{64} \mathrm{Ni}$ reactions indicates that transfer of nucleons affects the fusion process. The dashed lines show calculated cross sections within the one dimensional model.

\section{The various features of near- and sub-barrier heavy-ion fusion}

A wide range of interesting features have been shown by the studies of heavyion fusion reactions $[1,2]$. Indeed it was already observed in the experiments concerning ${ }^{16} \mathrm{O}$ on various $\mathrm{Sm}$ isotopes [3] and $\mathrm{Ni}+\mathrm{Ni}$ systems [4] that there is a strong interplay of dynamics and nuclear structure near and below the barrier as shown in Fig. 1.

The effect of coupling to transfer channels was revealed in several cases, and one of the most significant evidences was observed in the trend of the $\mathrm{Ca}+\mathrm{Zr}$ systems which show very different behaviours as reported in Fig. 2 (left panel). Here the fusion excitation functions have been plotted in a appropriate reduced energy scale to take into account the different Coulomb barriers and the high energy octupole vibrations of ${ }^{40} \mathrm{Ca}$ and ${ }^{48} \mathrm{Ca}[5,6]$. In this way, we highlight the effect of the neutron pick-up transfer channels that have positive Q-values only for ${ }^{40} \mathrm{Ca}+{ }^{94,96} \mathrm{Zr}$.

The concept of barrier distribution introduced in Ref. [7] was often successfully exploited to identify the nature of the coupled channels. In the 

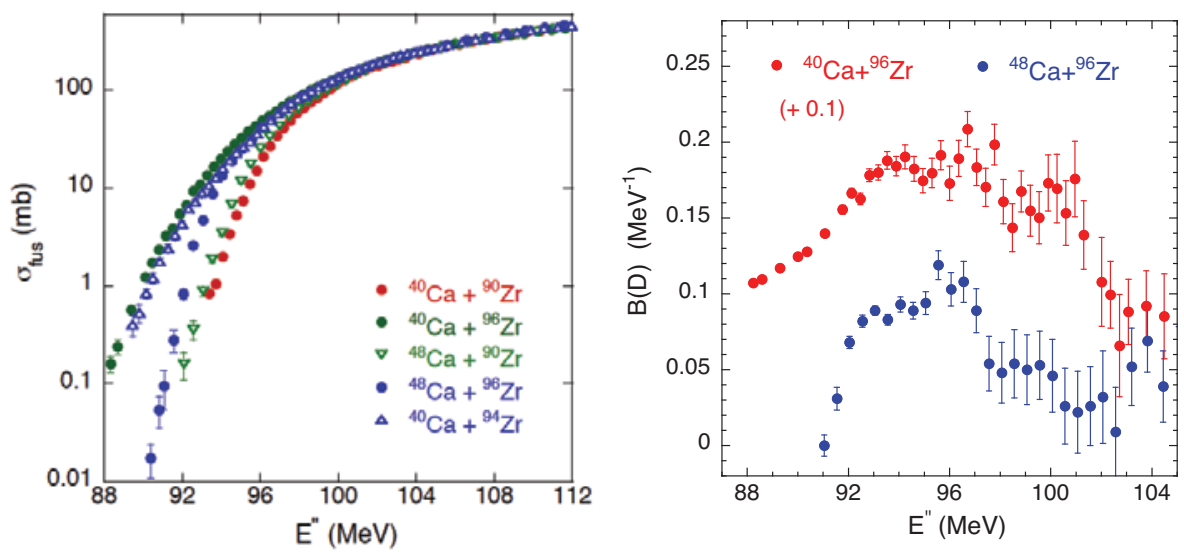

Figure 2: Left: Excitation functions for several Ca+Zr systems. Right: Barrier distributions for the two cases ${ }^{40,48} \mathrm{Ca}+{ }^{96} \mathrm{Zr}$.

examples reported in Fig. 2 (right panel) one can see that the effect of transfer channels is to produce a wider distribution with a tail towards low energies.

\section{Hindrance vs. enhancement}

In the energy region far below the Coulomb barrier fusion cross sections result from a balance between the enhancement produced by couplings which are dominant at energies close to the barrier, and the more recently observed hindrance phenomenon [8]. The left panel of Fig. 3 shows the excitation functions of the two systems ${ }^{48} \mathrm{Ti}+{ }^{58} \mathrm{Fe}[9]$ and ${ }^{58} \mathrm{Ni}+{ }^{54} \mathrm{Fe}[10]$. One notices the very large enhancement with respect to the no coupling limit and a fast change of slope for ${ }^{58} \mathrm{Ni}+{ }^{54} \mathrm{Fe}$ indicating the appearance of hindrance already at relatively large cross sections (energies), because these two nuclei are rather stiff. ${ }^{48} \mathrm{Ti}$ and ${ }^{58} \mathrm{Fe}$ are softer and no indication of hindrance is seen down to $\sim 1 \mu \mathrm{b}$.

A convenient way to show the trend of the low-energy excitation function is to plot the logarithmic derivative of the energy-weighted cross section $\mathrm{L}(\mathrm{E})$ (right panel of Fig. 3). Indeed the onset of hindrance is conventionally located at the energy where $\mathrm{L}(\mathrm{E})$ reaches the $\mathrm{L}_{C S}$ value which is expected for a constant astrophysical S factor [11]. The S-factor, originally introduced in nuclear astrophysics as a convenient representation for light-ion fusion reactions, shows a maximum at that energy. This is observed for ${ }^{58} \mathrm{Ni}+$ ${ }^{54} \mathrm{Fe}$, but not for ${ }^{48} \mathrm{Ti}+{ }^{58} \mathrm{Fe}$. 

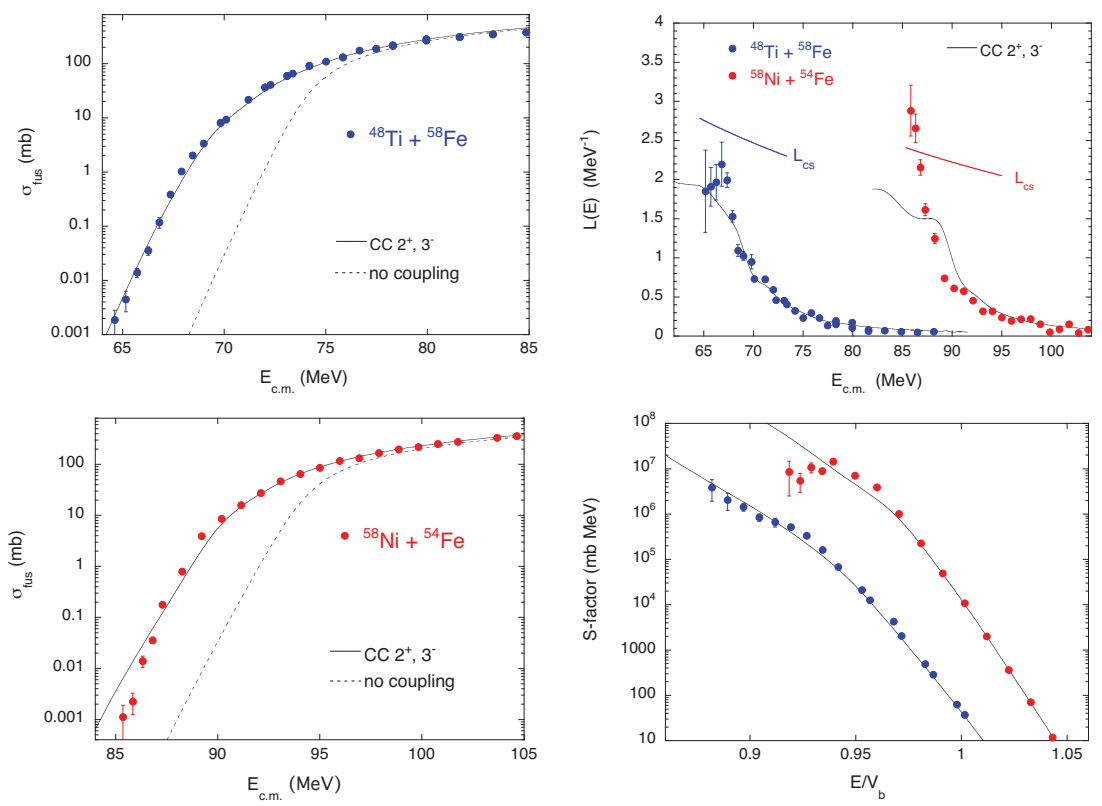

Figure 3: Excitation function of ${ }^{48} \mathrm{Ti}+{ }^{58} \mathrm{Fe}$ and ${ }^{58} \mathrm{Ni}+{ }^{54} \mathrm{Fe}$ (left panels), logarithmic derivatives and S-factors (right panels). Standard CC calculations do not reproduce the low energy ${ }^{58} \mathrm{Ni}+{ }^{54} \mathrm{Fe}$ data indicating the presence of hindrance.

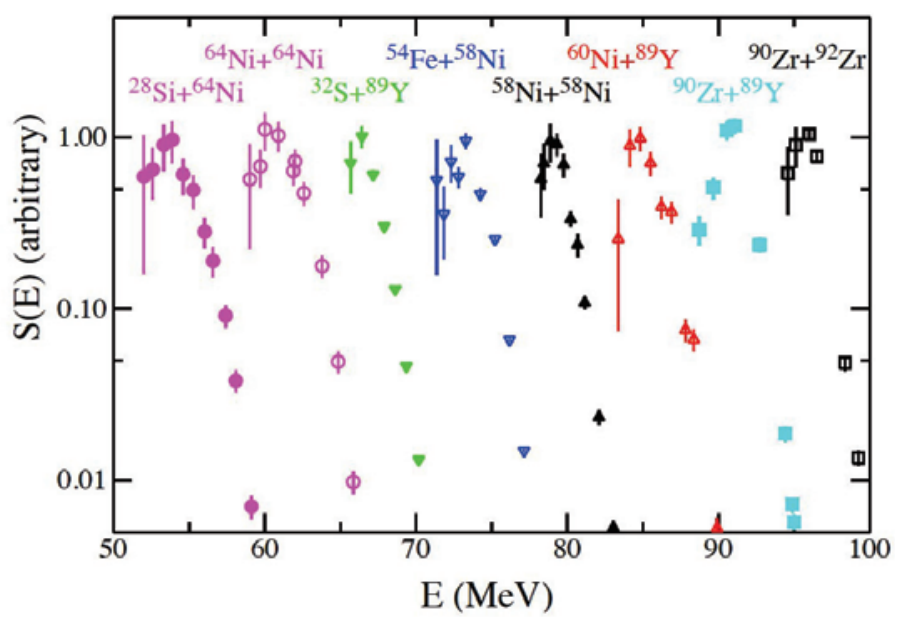

Figure 4: $S(E)$ for the systems whose cross sections at low energies are dominated by fusion evaporation and where maxima have been experimentally observed [12]. 

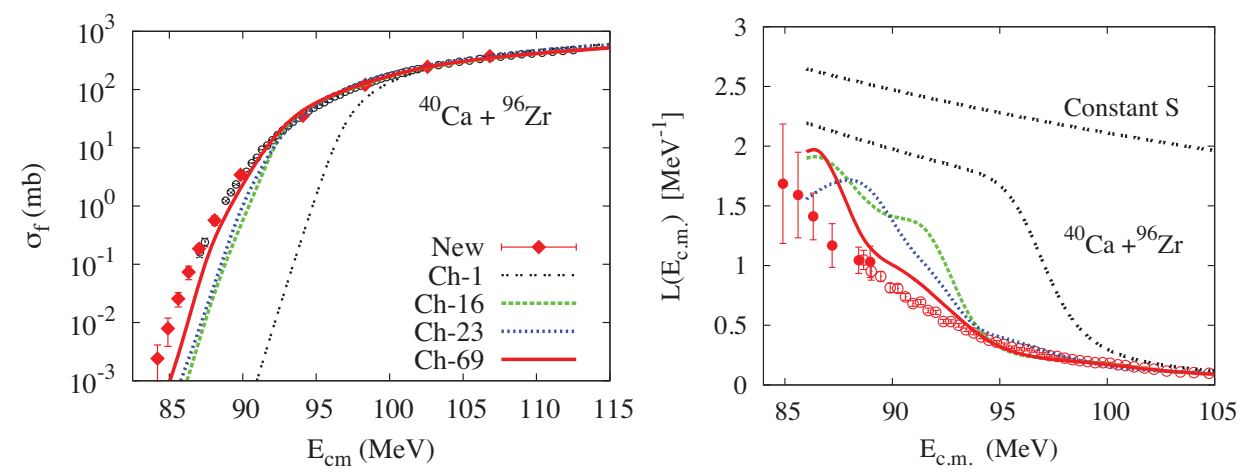

Figure 5: (Colour online) Left: Excitation function of ${ }^{40} \mathrm{Ca}+{ }^{96} \mathrm{Zr}$ in a logarithmic scale. The red and black symbols are the cross sections measured in Refs. [5, 13], respectively. The lines are the results of the CC calculations described in the text. Right: Slope $\mathrm{L}(\mathrm{E})$ of the fusion excitation function compared to the CC results.

Clear S-factor maxima have been detected in various cases (see Fig. 4 [12]). For other systems the hindrance is no so strong to produce a S-factor maximum and it is necessary to refer to a calculation in order to recognise that effect.

\section{Coupling to transfer channels}

Let us consider the special case of ${ }^{40} \mathrm{Ca}+{ }^{96} \mathrm{Zr}[5,13]$ where many neutron transfer channels with positive Q-values are available. Detailed CC calculations were performed [14] but even using a standard Woods-Saxon potential the fusion data at low energies are strongly underestimated and no indication of hindrance appears yet. The low-lying $2^{+}$and $3^{-}$states (Ch-28 in Fig. 5) produce strong effects. Couplings to $\mathrm{Q}>0$ one- and two-nucleon transfer channels (Ch-84) bring further significant enhancements, even at the level of a few $\mu \mathrm{b}, \mathrm{Ch}-1$ is the no-coupling limit.

This is confirmed when we plot the slope $\mathrm{L}(\mathrm{E})$ (right panel of Fig. 5), which remains very low with respect to the value expected for a constant $\mathrm{S}$ factor. In lighter cases as the various $\mathrm{Ca}+\mathrm{Ca}$ systems, analogous systematic trends have been observed. The effect of transfer is strong and clear for ${ }^{40} \mathrm{Ca}+{ }^{48} \mathrm{Ca}[15,16]$, whose cross sections exceed the ${ }^{48} \mathrm{Ca}+{ }^{48} \mathrm{Ca}$ data at low energies and are suppressed compared to the ${ }^{40} \mathrm{Ca}+{ }^{40} \mathrm{Ca}$ data at high energies. In spite of the large enhancement in the sub-barrier fusion of ${ }^{40} \mathrm{Ca}+{ }^{48} \mathrm{Ca}$, a hindrance does eventually occur but the onset is pushed down to very low energies, where $\mathrm{L}(\mathrm{E})$ increases rapidly. 

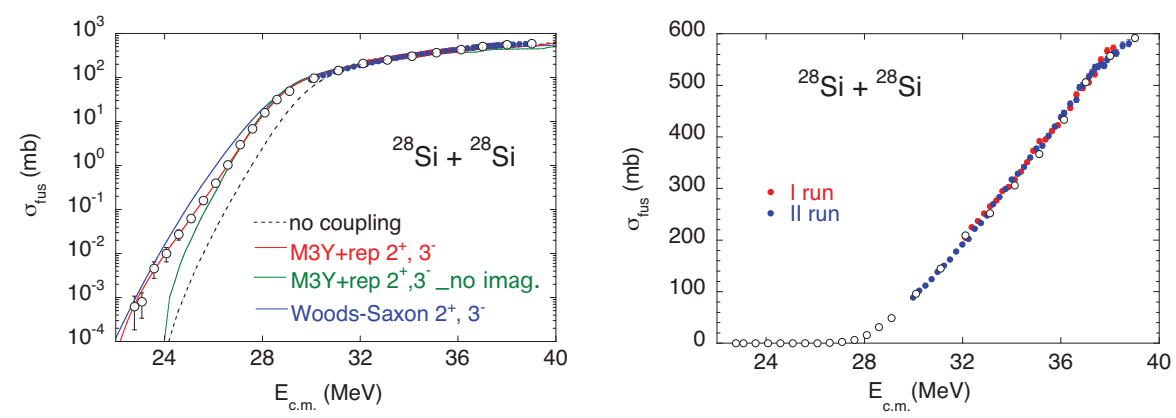

Figure 6: Fusion excitation function of ${ }^{28} \mathrm{Si}+{ }^{28} \mathrm{Si}$ in logarithmic (left) and linear (right) scales $[22,23]$. The results of the CC calculations discussed in the text are also shown in the left panel.

\section{The case of the ${ }^{28,30} \mathrm{Si}+{ }^{28,30} \mathrm{Si}$ systems}

The excitation functions of various light heavy-ion systems indicate that oscillatory structures appear above the barrier, probably due to the penetration of successive centrifugal barriers [17]. A good example for this is the case of ${ }^{16} \mathrm{O}+{ }^{16} \mathrm{O}[18,19]$. A shallow potential is needed to fit the data above (as well as below) the barrier [20]. More recently TDHF calculations have also been performed to reproduce those oscillations [21].

It is very interesting to investigate heavier systems, where the sub-barrier hindrance phenomenon is stronger and better established. A convincing observation of such oscillations may provide useful information on the ionion potential in a wide energy range. Therefore we recently measured the fusion excitation function of ${ }^{28} \mathrm{Si}+{ }^{28} \mathrm{Si}$ from below to above the barrier with very small energy steps in the higher energy region, please see Fig. $6[22,23]$. In the left panel of this figure the results of CC calculations are shown, including the low-lying $2^{+}$and $3^{-}$states, and their mutual excitations. Using a standard Woods-Saxon potential, the calculation overpredicts the data just below the barrier but the fit improves at the lowest energies.

On the other hand by employing the M3Y shallow potential (see the green line) the data are clearly underestimated below the barrier. To reproduce the experimental data a weak and short-ranged imaginary potential $\left(\mathrm{W}_{0}=5 \mathrm{MeV}, \mathrm{a}=0.2 \mathrm{fm}\right)$ is required at low energies, that possibly simulates the effect of the strong oblate deformation of ${ }^{28} \mathrm{Si}$.

Using the first derivative of the excitation function $d(E \sigma) / d E$ makes it easier to observe the oscillations [20]. The first derivative obtained from the detailed cross section measurement above the barrier shows distinct 


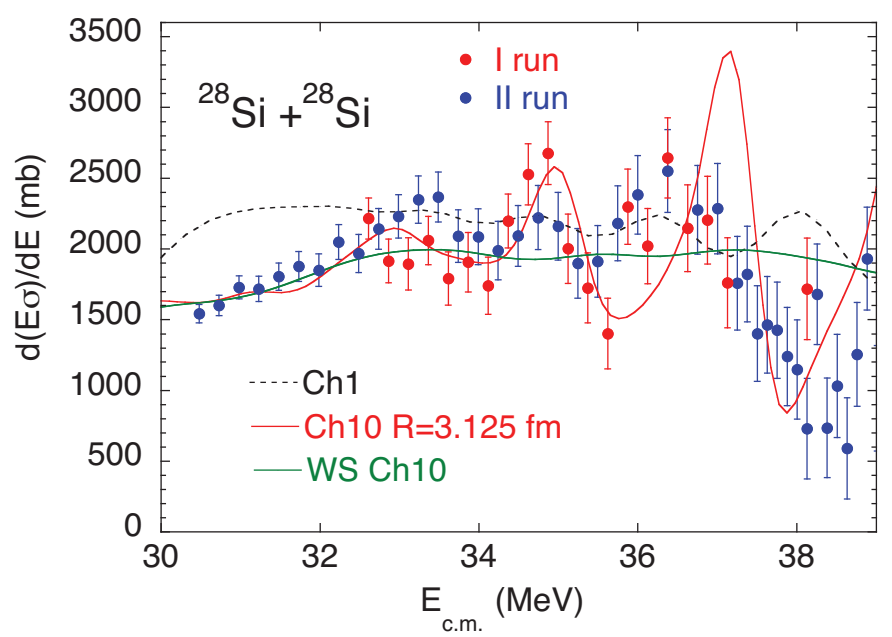

Figure 7: The derivative of the energy-weighted fusion excitation function $d(E \sigma) / d E$ of ${ }^{28} \mathrm{Si}+{ }^{28} \mathrm{Si}$ in an energy range above the barrier. The derivative is obtained as the incremental ratio between successive points, with an energy step $\Delta \mathrm{E}_{c m}=0.75 \mathrm{MeV}$. The lines are the results of the theoretical calculations discussed in the text.
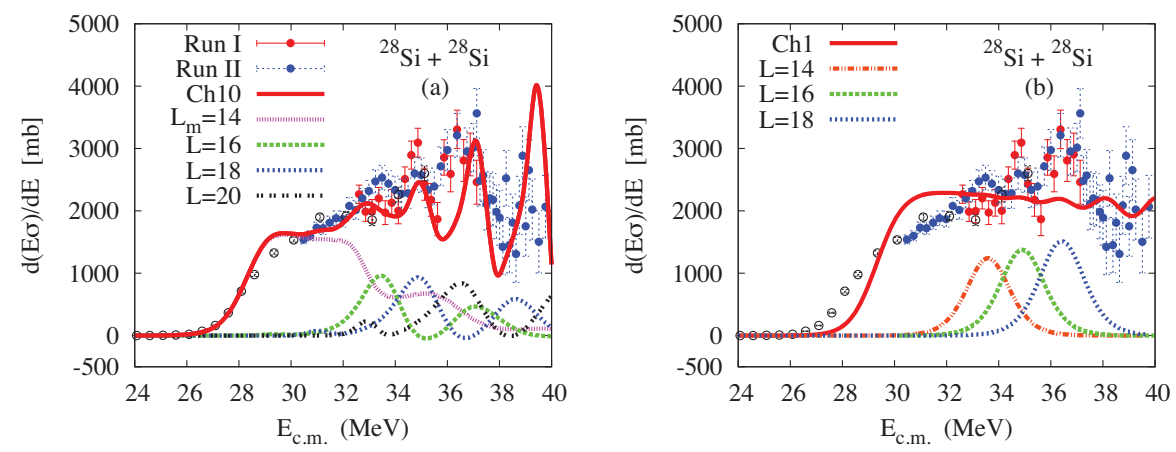

Figure 8: (Colour online) (a) The first derivative of the excitation function is compared to the results of Ch10 calculations. The red (magenta) curve is the result using a maximum angular momentum for fusion $L_{m}=38\left(L_{m}=14\right)$. The individual contributions for $L=16-20$ are also reported. (b) The results of Ch1 calculations are shown. The experimental peaks at 33,35 , and $37 \mathrm{MeV}$ are associated with $L$ $=14,16$, and 18 , while they are primarily related to $L=16,18$, and 20 in Ch10 calculations shown in panel (a). 

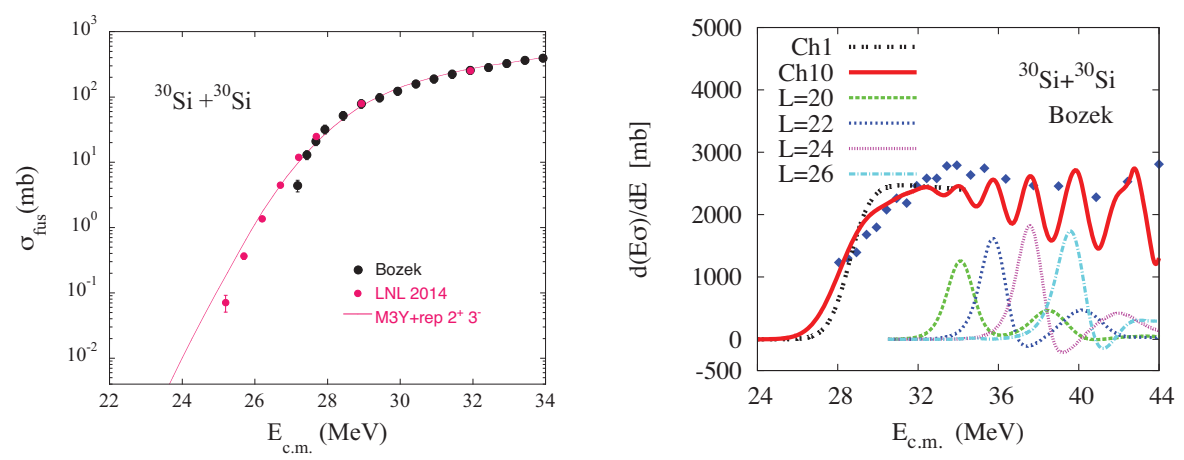

Figure 9: (Colour online) Left: Excitation function of ${ }^{30} \mathrm{Si}+{ }^{30} \mathrm{Si}$ as measured by Bozek et al. [24], with the few additional (preliminary) points recently measured at LNL. The red line is the early CC calculation cited in the text. Right: The derivative obtained from the data of Bozek et al. (blue points) is shown together with the CC results (red line) predicting well defined oscillations.

oscillations although the uncertainties are rather large, as reported in Fig. 7. This behaviour has been confirmed in two different series of measurements ( I run and II run in Fig. 7). In that figure the comparison with the CC calculations using M3Y+repulsion potential, have been shown. The Ch1 is the no-coupling limit and Ch10 is the result when the $2^{+}$and $3^{-}$states are considered. One can notice that only by including the channel couplings, a reasonable fit of the data is obtained.

The calculation has also been performed employing the Woods-Saxon potential but the result is not equally satisfying (WS Ch10 in the Fig. 7) because very weak oscillations show up, providing a poor fit to the data.

Although the channel couplings are essential to reproduce the data, they split the contribution of a single L (see Fig. 8(a)) so that the correspondence of the experimental peaks to the penetration of specific centrifugal barriers is lost. Fig. 8(b) reports the results of no-coupling calculations (Ch1) where the oscillations are smoothed out because the contributions to the single Lvalues (reflecting the individual centrifugal barriers) produce wider peaks.

We have undertaken very recently the study of ${ }^{30} \mathrm{Si}+{ }^{30} \mathrm{Si}$ because this nucleus has a spherical shape. Therefore it can be a good reference point to better understand the role of the oblate deformation of ${ }^{28} \mathrm{Si}$. The Fig. 9 (left) shows the excitation function of ${ }^{30} \mathrm{Si}+{ }^{30} \mathrm{Si}$ near and below the barrier as measured by Bozek et al. [24] and, very recently, at LNL. The result of preliminary CC calculations is also shown, but the data have to be completed with more detailed measurements. 
An experiment aiming at the measurement of oscillations in this system will be performed at LNL very soon, where oscillations have been predicted (see Fig. 9 (right)). The coupling to the low-lying states is weaker than in the case of ${ }^{28} \mathrm{Si}+{ }^{28} \mathrm{Si}$, so that the oscillatory structures, if confirmed, could be associated to the individual centrifugal barriers as for lighter systems. The derivative extracted from the existing data [24] is reported in the figure but the very large errors (not shown here) do not allow any conclusion.

\section{Summary}

Sub-barrier fusion cross sections are strongly enhanced by couplings of the relative motion to nuclear shape deformations and vibrations $[1,2]$. Analogous effects due to nucleon transfer degrees of freedom have been searched for, following the early experiments for the various $\mathrm{Ni}+\mathrm{Ni}$ systems [4], but the role of nucleon transfer has been unambiguously identified only in few other cases. The discovery of the hindrance phenomenon [8] at very low energies, has made the situation more interesting but even more complex. Indeed, fusion cross sections are determined by the concurring contributions of hindrance and enhancement in that energy range where transfer couplings are expected to be still effective.

The appearance of oscillations and the trend of sub-barrier cross sections in ${ }^{28} \mathrm{Si}+{ }^{28} \mathrm{Si}$ have been reproduced within the same theoretical frame, i.e., the CC model using the shallow M3Y+repulsion potential. Within that model the existence of oscillations is tightly bound to channel couplings, while in lighter cases (e.g. ${ }^{16} \mathrm{O}+{ }^{16} \mathrm{O}$ ) the oscillations are related to the overcoming of successive centrifugal barriers well spaced in energy. As a consequence, the one-to-one relation between each peak and the height of a centrifugal barrier is lost in ${ }^{28} \mathrm{Si}+{ }^{28} \mathrm{Si}$. The nature of these oscillations and the behaviour of the sub-barrier excitation function might be clarified by analogous measurements for ${ }^{30} \mathrm{Si}+{ }^{30} \mathrm{Si}$ because ${ }^{30} \mathrm{Si}$ is a spherical nucleus while ${ }^{28} \mathrm{Si}$ has a permanent oblate deformation. Such experiments on ${ }^{30} \mathrm{Si}+{ }^{30} \mathrm{Si}$ are in progress at LNL.

My grateful thanks go to all my colleagues who made possible to perform the research and to obtain the results reported in this paper.

\section{References}

[1] M.Dasgupta, D.J.Hinde, N.Rowley and A.M.Stefanini, Annu. Rev. Nucl. Part. Sci. 48, 401 (1998). 
[2] B.B.Back, H.Esbensen, C.L.Jiang and K.E.Rehm, Rev. Mod. Phys. 86, 317 (2014).

[3] W.Reisdorf et al., Nucl. Phys. A 438, 212 (1985).

[4] M.Beckerman et al., Phys. Rev. Lett. 45, 1472 (1980).

[5] A.M.Stefanini et al., Phys. Lett. B 728, 639 (2014).

[6] A.M.Stefanini et al., Phys. Rev. C 76, 014610 (2007).

[7] N.Rowley, G.R.Satchler and P.H.Stelson, Phys. Lett. B 254, 25 (1991).

[8] C. L. Jiang et al., Phys. Rev.Lett. 89, 052701 (2002).

[9] A.M.Stefanini et al., EPJ Web of Conf. 86, 00057 (2015).

[10] A.M.Stefanini et al., Phys. Rev. C 81, 037601 (2010).

[11] C.L.Jiang et al., Phys. Rev. Lett. 93, 012701 (2004).

[12] C.L.Jiang, private communication.

[13] H.Timmers et al. Nucl. Phys. A 633, 421 (1998).

[14] H.Esbensen and A.M. Stefanini, Phys. Rev. C 89,044616 (2014).

[15] G. Montagnoli et al., Phys. Rev. C 85, 024607 (2012).

[16] C. L. Jiang et al., Phys. Rev. C 82, 041601(R) (2010).

[17] N.Poffé, N.Rowley and R. Lindsay, Nucl. Phys. A 410, 498 (1983).

[18] I.Tserruya et al., Phys. Rev. C 18, 1688 (1978).

[19] J.Thomas et al., Phys. Rev. C 33, 1679 (1986).

[20] H.Esbensen, Phys. Rev. C 85, 064611 (2012).

[21] C.Simenel et al., Phys. Rev. C 88, 024617 (2013).

[22] G.Montagnoli et al, Phys. Rev. C 90, 044608 (2014).

[23] G.Montagnoli et al., Phys. Lett. B 746, 300 (2015).

[24] E. Bozek et al., Nucl. Phys. A 451, 171 (1986). 